\title{
LOS FUNDAMENTOS DISCURSIVOS DE LA DEMOCRACIA Y LOS CONFLICTOS DE INTERESES
}

OSVALDO GUARIGLIA

Centro de Investigaciones Filosóficas, CONICET

Universidades de La Plata y Buenos Aires

1. El problema que me propongo tratar es sólo parcialmente aludido por el título de mi contribución. Pretendo establecer dentro de qué límites teóricos estamos hablando cuando pretendemos 'fundamentar' la democracia y qué alcance, por consiguiente, puede tener esta 'fundamentación' dentro del complejo fenómeno político al que globalmente designamos bajo este título. A tal efecto, haré primero algunas disquisiciones semánticas en torno a los varios significados que posee el término 'democracia'; estableceré, de inmediato, tres esferas distintas de acción en cada una de las cuales el término remite a un tipo diferenciado de acciones posibles; delimitaré, luego, dentro de cuáles de estas esferas es posible echar, al menos tentativamente, los fundamentos de la democracia y examinaré algunas estrategias seguidas con ese fin; propondré, de inmediato, mi propia estrategia y los límites de su alcance, por último, conectaré estas limitaciones con las dificultades que se presentan dentro de una realidad sumamente conflictiva en los estadios designados como de 'transición' hacia la democracia.

2. A fin de convencernos de que el vocablo 'democracia' es esencialmente equívoco, basta con echar una mirada a los distintos regímenes cuya estructura política interna se halla, en algunos casos, en extremos opuestos y que, sin embargo, ostentan a veces, en su misma designación como estado nacional, el término democracia. Sería, a mi juicio, tarea vana pretender encontrar un denominador común que nos revelara algún rasgo permanente con el que podríamos identificar la característica distintiva de este régimen. A cambio de ello, es conveniente partir de un análisis conceptual de los usos del término en distintos contextos. De esta manera es posible diferenciar tres usos posibles en contextos (a) evaluativos, (b) normativos y (c) descriptivos...

Ejemplo de (a): "el estado X es una democracia, no una aristocracia"; ejemplo de (b): "para que X fuese una democracia, debería reconocer el derecho de los más pobres al voto"; y de (c): "en una democracia existen diversos partidos políticos". Por cierto, los límites entre uno y otro usos son difusos y quedan 
abiertos a la controversia. Creo, sin embargo, que la distinción que acabo de hacer no solamente está metódicamente fundada sino que es fructífera en el análisis de las cuestiones conceptuales que se nos presentan en el tratamiento del tema. En términos generales, hay envueltos, a mi modo de ver, criterios evaluativos en toda distinción entre dos estados posibles de una misma sociedad, o entre dos sociedades, tal que uno o una de ellos sea calificado como 'democrático' y el otro o la otra como 'aristocrático', 'oligárquico', 'totalitario', etc. Esta distinción evaluativa se diferencia del aspecto normativo, el cual, en términos generales, establece los requisitos que en cada caso se consideran necesarios a fin de que un determinado régimen pueda ser calificado como 'democrático'. Estos requisitos se expresarán bajo la forma de normas que involucran a los miembros de una determinada sociedad en un determinado momento de su evolución histórica. Por último, el aspecto descriptivo supone la preeminencia de un estado fáctico de relaciones que se han mantenido ininterrumpidamente durante un considerable espacio de tiempo, el cual goza de reconocimiento general por parte de los participantes del régimen político en relación con los requisitos exigidos a un régimen democrático, los que se consideran satisfechos. En ese estado de relaciones el término 'democracia' describe un corpus jurídico y un conjunto de hábitos y prácticas sociales que tienen efectiva existencia.

Cada uno de estos aspectos, el evaluativo, el normativo y el descriptivo, que son claramente diferenciables en el significado del término, remite en última instancia a una esfera propia y autónoma de acción, que distinguimos así: (a) evaluativa, (b) práctico-normativa y (c) pragmática. La primera está constituida por estados de relaciones entre los miembros de una misma comunidad; la segunda, por normas universales que involucran a los miembros de una comunidad en tanto sujetos humanos; la tercera y última, por interacciones de los miembros de una comunidad en tanto agentes con la naturaleza tanto la nuestra propia como la que nos rodea; la dificultad más grande con la que topamos al precisar el sentido de 'democracia', consiste, justamente, en el irremediable entrecruzamiento de sentidos que remite a cada una de estas tres esferas, entrecruzamiento que, al no ser claramente analizado, obstaculiza seriamente el mutuo entendimiento.

Esta situación ha conducido a que, en la mayoría de los casos, se haya escogido una sola de las tres significaciones posibles, haciendo de ella la significación esclusiva de 'democracia' y relegando a las otras a meros aspectos secundarios y subordinados.

Para una concepción evaluativa como, por ejemplo, la de Mill o la de Marx, 'democracia' designa solamente un determinado estado de relaciones con referencia al cual obtendrán todos los otros estados posibles de relaciones, calificaciones en una escala gradual según su probable cercanía o lejanía a él. Considerada de esta manera, la democracia en la esfera normativa pasa a convertirse 
en una mera regulación formal, de carácter técnico, en el mismo nivel que la esfera pragmática. En efecto, tanto la tradición cultural, histórica y jurídica de una sociedad, que determina el nivel de conciencia moral y el estatus jurídico de sus miembros, como la riqueza del suelo y el grado de evolución técnica y científica de los habitantes, son datos de la realidad que deben ser tenidos en cuenta solamente como medios en función del fin constituido por el estado de relaciones presentado como meta posible a alcanzar.

En cambio, es evidente que para una concepción práctico-normativa de democracia, tanto los estados de relaciones entre los miembros de una sociedad, que en última instancia dependen de determinadas características naturales de sus miembros y de la relación entre ellos, como las condiciones naturales, económicas, técnicas y sociales, en las que se despliega el juego democrático, asumen un papel secundario, en la medida en que proveen solamente las condiciones empíricas en sentido amplio con las que deberá enfrentarse y a las que deberá restringirse el modelo normativo ideal.

Por último, para una concepción pragmática, 'democracia' se redefine como un término puramente descriptivo que hace referencia al funcionamiento de un proceso de selección entre opciones de acción colectiva fundamentalmente en el ámbito de la economía y, consecuentemente, en el de la distribución del producto social.

3. En un trabajo anterior ${ }^{1}$ he argumentado en el sentido de que estas tres significaciones posibles de 'democracia' que remiten a tres ámbitos de acción distintos, evaluativo, normativo y pragmático, son congruentes con la existencia de tres esferas diferenciadas y autónomas de acción social en la moderna sociedad. Tal ha sido, como es notorio, el mérito de la obra de $M$. Weber, especialmente su reconstrucción del advenimiento de la moderna sociedad occidental como un proceso de progesiva racionalización e independización de los distintos tipos de acciones, originariamente no diferenciados. Los estudios de W. Schluchter, J. Habermas y otros ${ }^{2}$ han aportado, por una parte, una mayor claridad sobre la base conceptual en la que Weber se apoyaba, y por otra, han puesto de manifiesto la necesidad de ir más allá que Weber en la reconstrucción lógico-sistemática de estos tipos de acción. Apelando a una pragmática de los actos linguiísticos, Habermas ha propuesto reestructurar los tipos de acción social en tres grandes categorías: la acción instrumental, que coincide en general con la acción con respecto a un fin de Weber, la acción normativa, que corresponde en parte a la racionalidad valorativa de Weber, $y$, por último, la acción expresiva, de la que no hay correlato explícito en este último. 3

1 Comp. Guariglia, $P C D$, pp. 35 ss.

2 Comp. Guariglia, $I V L$, pp. 221 ss.

3 Comp. Habermas, TKH I, pp. 126 sa, 148 ss. 
Esta división de Habemas presenta, a mi juicio, serias dificultades, para establecer el lugar sistemático tanto de los términos valorativos en el lenguaje como de la vigencia de los valores positivos en las acciones. En efecto, como creo poder demostrar en otro trabajo, Habermas no distingue suficientemente entre normas y valores, con lo que un ámbito sumamente importante de la acción moral y política queda seriamente desdibujado. Frente a ésta, mi propuesta retiene la distinción entre una esfera de acción instrumental o pragmática, cuya estructura es técnica y está dirigida a producir cadenas causales entre los medios disponibles y los fines propuestos, una esfera de acción normativa o jus-práctica, constituida exclusivamente por las normas universales de carácter moral o jurídico, y por último una esfera de acción evaluativa o estético-práctica, en la que se desarrollan las acciones tendientes a producir o corporizar valores positivos especialmente apreciados por las diversas comunidades sociales, en las diversas épocas históricas. ${ }^{4}$

Pues bien, puestas en correlación las tres significaciones de 'democracia' antes discutidas con estas tres posibles esferas sociales y políticas de acción, ¿a cuál de ellas nos referimos al hablar de "los fundamentos de la democracia"? ¿No es posible, en última instancia, que en cada una de ellas haya una clase propia de fundamentos como condiciones sine qua non de tal régimen político? Por cierto, creo que la respuesta es, en parte, afirmativa y que, en cierto modo, nos encontramos aquí frente al dilema kantiano de que deber implica poder. En efecto, dado que, por un lado, tanto la acción técnicopráctica, en la medida en que supone un saber teórico de las relaciones causales entre las cosas, como la acción práctico-estética, en la medida en que anticipa estados posibles sea de los seres humanos entre sí sea de su relación productiva o realizativa con la naturaleza, ambos tipos de acción se remiten a una realidad actual. La acción jus-práctica, en cambio comprende a todos los enunciados vigentes de deber ser en sentido moral o legal estricto, y por lo tanto, no tiene contacto estructural alguno con la realidad actual, a la que sin embargo, supone como condicionante. Pues, sin duda, es evidente que debe haber determinada organización social y económica y cierta historia anterior de las condiciones de producción, organización y distribución del trabajo y del producto social así como la permanencia de ciertas regulaciones técnico-funcionales existosas en el mantenimiento del sistema como condición pragmática de la democracia. Pero es también evidente que estas condiciones tienen que ser congruentes de un modo o de otro con los ideales de vida personal, comunitario, social, religioso o estético de los miembros de la sociedad, para que el sistema aludido funcione espontáneamente y sin resistencias insuperables.

4 Una argumentación más detallada de esta propuesta se encontrará en Guariglia, "Razón práctica e intereses de la acción”, Diánoia 33 (1987), pp. 53-68. 
Ahora bien, en lo que acabo de afirmar está ya, a mi entender, la resolución del problema. En efecto, cuando hablamos de 'fundamentos' en el sentido empleado más arriba, entendemos claramente por ello determinadas razones últimas de una determinada conducta, razones, que por definición, dependen exclusivamente de nosostros mismos, es decir, están, si están, a nuestra disposición para decidir una determinada forma de actuar. Por lo tanto, dado que tanto la acción pragmática como la práctico-estética tienen una indisoluble relación con la realidad exterior, sea natural en el primer caso, sea, además de la natural, la histórico-cultural en el segundo, no podemos hablar con relación a ellos de 'fundamentos' de un tipo de conducta colectiva, como es la democracia, sino de 'condiciones', que, dado su carácter fáctico, se dan en mayor o menor grado o, simplemente, no se dan.

En conclusión, cuando hablamos de 'fundamentos' de la democracia, estamos reconociendo implícitamente una determinada primacía lógica a la esfera normativa de significación y al correspondiente tipo de acción jus-práctica frente a las otras dos. En este ámbito de acción y de racionalidades es en el que debemos buscar los fundamentos tras los que vamos.

4. Asumir como tarea los búsqueda de los fundamentos de la democracia equivale, como señalé antes, a seleccionar aquellas razones últimas que justifiquen la adopción de una forma de acción e interacción, por el conjunto de los miembros de la sociedad, normada de una determinada manera y que esa adopción sea en lo sucesivo respetada. Por tanto, debemos buscar los fundamentos en alguna forma de racionalidad apropiada para este tipo de acción, es decir, una racionalidad práctica. Tan pronto como enunciamos el objetivo, se presenta un sinnúmero de objeciones a él. Como es sabido, no sólo se ha negado (desde $\mathrm{D}$. Hume en adelante) la existencia de una razón práctica que promueva acción alguna, sino que la misma razón teórica, cuya existencia no es puesta en duda por la tradición no-cognitivista en materia moral, ha sido y es fuertemente relativizada como facultad universal, es decir, como facultad que sea atribuible, en principio, a todos los hombres de todas las culturas en todas las épocas de la misma manera. En especial, esta relativización ha provenido del campo de la antropología cultural al confrontar nuestra actual civilización con prácticas rituales, creencias mágicas y representaciones animistas del mundo que resultaban completamente irracionales de acuerdo con nuestros criterios actuales de evaluación. ${ }^{5}$

La noción de una razón universal es puesta, de este modo, fuertemente en duda, y aparece sustituida por la noción de lo que en cada caso se tiene por razón o está implícito como razón. De este modo, ningún tipo de racionalidad podría trascender el nivel de prácticas primarias y de términos lingüísticos

5 Comp. la recopilación de las contribuciones más importantes de ese debate hecha por $\mathrm{B}$. R. Wilson (ed.), Rationality, Blackwell, Oxford, 1984 (la. ed., 1970). 
en las que aparece corporizada, ya que toda generalización más allá de estos límites cometería una distorsión destructiva de la idiosincracia del concepto en cuestión. ${ }^{6}$ Como puede verse, ha sido éste, sin duda, el modelo de una razón particularizada en determinadas culturas y regiones que ha penetrado profundamente en ciertas corrientes del pensamiento político contemporáneo, muy especialmente en América Latina.

Habiendo conducido la concepción sustancialista de razón como autoconciencia, que fue propia de la filosofia alemana de Kant hasta nuestros días, ${ }^{7}$ a un callejón sin salida y habiendo hecho crisis, en consecuencia, la noción de facultad global que estaba en su base, debemos hoy contentarnos con sustitutos mucho más modestos a fin de poder fijar algunos pocos rasgos comunes que caractericen tal facultad. Tal ha sido fundamentalmente en los últimos tiempos la noción wittgensteiniana de "seguir una regla" como capacidad demostrativa de una determinada competencia. La reflexión, en este caso, se lleva a cabo sobre determinadas formas de comunicación y de comportamiento lingüístico que están previamente dados a todos los sujetos hablantes y actuantes de una cierta comunidad. La noción de racionalidad práctica que voy a adoptar aquí como válida, es la que emerge implícita en determinadas reglas pragmáticas del uso de actos lingüísticos como una lógica propia de todos los hablantes que hacen uso de un cierto lenguaje, en mi caso el español. Con ello admito, con Habermas, el carácter paradigmático que asume la reconstrucción abstracta de un sistema de reglas como modelo formal e ideal, por contraste con el cual podemos juzgar críticamente la realidad empírica. ${ }^{8} \mathrm{Si}$ es posible una respuesta a la actitud relativista con relación a la razón y, muy especialmente, a la razón práctica, creo que éste es el camino que más promete. Al menos, evita de entrada un error que se le ha señalado al constructivismo de tipo rawlsiano, el cual es el de desechar el uso ordinario del lenguaje como guía en la reconstrucción lógica de los juicios éticos, con lo que luego la retraducción de los juicios constructivos a juicios habituales se hace más que problemática. Lo que estoy cuestionando no es, por cierto, los puntos centrales de la concepción rawlsiana de justicia (con la que, como se verá, mi propia propuesta tiene algunos puntos de contacto), sino la estrategia seguida en la fundamentación de la validez de sus principios.

5. Voy a señalar los límites modestos que propongo para el tipo de fundamentación racional en el campo normativo que quiero introducir. En primer

6 Comp. P. Winch, en Rationality, pp. 10 ss.

7 Comp. E. Tugendhat, Vorlesungen zur Einfuibrung in die Sprachanalytische Philosophic, Suhrkamp, Francfort, 2a. ed., 1976, pp. 95-104. Pp. 28-29. 
lugar, si bien apelo a reglas pragmáticas que rigen una clase de actos ilocucionarios, no les doy a estas reglas ningún carácter trascendental sino solamente reflexivo, reconstructivo y, como tal, están basadas en procedimientos empíricamente constatables. En segundo lugar, la existencia de tales reglas no constituye, eo ipso, un fundamento coercitivo inapelable de tipo normativo, tal como sostiene Apel. En cierto modo no hay posibilidad de fundamentar de este modo la ética, porque sería de algún modo un tipo de derivación de normas a partir de hechos. Creo, en cambio, que lo que el conjunto de reglas provee es un modelo, un prospecto a seguir de comportamiento normativo fundado. Dado que, en última instancia, una vez reconocido como sistema de reglas, se trata de un conocimiento inmediato, la fuerza persuasiva de este conocimiento puede asimilarse a una coerción de la razón. Por último, las reglas pragmáticas de carácter reflexivo que voy a desarrollar con respecto a una clase de actos lingüísticos, los asociativos, no son, tampoco, el fundamento que busco, sino que conducen a este fundamento, que está constituido por los principios desarrollados a través de estas reglas.

Habermas ha pretendido reducir las clases de actos ilocucionarios a tres, que corresponden a sus tres tipos de acción: instrumentales, normativas y expresivas. Su intento, sin embargo, como él mismo acepta, ${ }^{9}$ está sujeto a revisión sobre la base de una discusión detallada de sus diversos aspectos. No creo conveniente, por ello mismo, tomar la clasificación de Habermas de actos lingüísticos, sino partir de la que propone J. Searle, ${ }^{10}$ que está simplemente orientada a introducir un cierto orden lógico en un rico material empírico. Searle toma como criterios de clasificación los siguientes: el objetivo ilocucionario, la dirección de la relación entre la proposición y el mundo, las condiciones de sinceridad y el contenido proposicional. Utilizando estos criterios, Searle distingue cinco categorías de actos lingüísticos: asertivos, directivos, compromisivos, expresivos y declarativos. De entre ellos, sólo dos tiene relación con una posible fundamentación de las normas: los directivos, cuyo objetivo ilocucionario consiste en el hecho de que son tentativas del hablante de obtener algo del oyente, y los compromisivos, cuyo objetivo ilocucionario consiste en comprometer al hablante en un cierto curso de acción futura. La simbolización de ambos es la siguiente:

Directivos: ! $V(O$ hace $A)(V=$ voluntad $)$

Compromisivos: $P \uparrow I(H$ hace $A)(I=$ intención $)$

Ahora bien, si observamos ambos tipos de actos desde el punto de vista de una fundamentación posible de normas, es claro que existe en ambos un

9 Comp. Habermas, TKH I, p. 433.

10 Comp. Searle, LA, pp. 9 ss. 
déficit. En efecto, a partir de los directivos podríamos llegar un tipo de fundamentación que considera a las normas como puras prescripciones, semejantes a los imperativos, dirigidos a la segunda persona, de tal modo que una universalización posible de este procedimiento nos conduciría, a lo sumo, a una propuesta de universalizabilidad como la de R. M. Hare. Como ha mostrado C. C. W. Taylor ya en la reseña de Freedom and Reason, tal clase de universalizabilidad es insuficiente para descartar comportamientos extremos como los de un racista. Si, en cambio, tomamos la clase de los compromisivos, como en algún momento ha hecho Apel, es evidente que por esta vía se puede comprobar la validez moral, en el sentido de un acto universalizado, de actos como promesas, pedidos de préstamos, y hasta casos como el famoso del depósito de Kant, dado que están involucrados marcos institucionales que obligan al hablante, pero a su vez, este compromiso no es simétrico por parte del oyente. De tal modo que no está dado tampoco aquí una base de fundamentación discursiva que comprometa a ambos participantes del diálogo a la vez. Existe, sin embargo, una clase de actos lingüísticos, no distinguida ni por Searle ni por Habermas, que ofrece esta posibilidad. Es posible que determinadas características sintácticas del inglés y del alemán hayan oscurecido la existencia de esta clase, que creo que es bien diferenciada en el español y otra lenguas romances. Llamo a estos actos asociativos, y su objetivo ilocucionario consiste en lograr que el hablante y el oyente realicen juntos un determinado curso de acción, al cual el hablante se compromete si el oyente acepta.

Simbolización: $E$ ? $\uparrow I V(H+O$ hacen $A)$

El objetivo ilocucionario es simbolizado $E$ ? porque constituye el núcleo performativo de las exhortaciones. Lo que distingue a éstas de los actos directivos es, justamente, este aspecto de invitación abierta que no puede ser satisfecha, si no es por medio de una aceptación explícita del oyente.

Además, en los actos asociativos no se trata de lograr sólo que el oyente haga algo, sino que expresan al mismo tiempo el compromiso del hablante de hacer lo mismo. La flecha es, en consecuencia, la misma que para los directivos y compromisivos, del mundo a las palabras. Las condiciones de sinceridad son las mismas que para los directivos y compromisivos a un tiempo: intención y voluntad. El contenido proposicional es que el hablante y el oyente adopten para futuro un determinado curso de acción. Algunos de los verbos de este grupo son: 'convenir', 'acordar', 'ponerse de acuerdo', 'discutir' (en algunos de sus usos), 'pactar'.

Ahora bien, la estructura sintáctica de los actos asociativos tiene en español ( $y$ otra lenguas romances) un rasgo peculiar que constituye un signo distintivo: el uso del subjuntivo presente en la oración principal. "Convengamos en que no nos agrediremos más"; "pongámonos de acuerdo en que elaboraremos ambos la política económica"; “acordemos tratar algunos de estos temas 
en las sesiones extraordinarias"; “discutamos sí, qué, cómo, etc.”; “pactemos si vamos a jugar uno o varios partidos”. La interrogativa indirecta (sí, qué, etc.) es una clara proposición objetiva. Esto se hace evidente en el uso ppdo: pactaron, acordaron, convinieron que... Estructura general: Yo/(Tú) verbo subjuntivo la. persona plural + Tú/(Yo) verbo futuro.

6. Una vez introducidos los actos asociativos, deseo poner en claro desde el comienzo qué me propongo lograr mediante el empleo de una instancia de ellos. Lo que pretendo hacer es restringir el postulado abierto de una ética discursiva como fundamento de las normas, que deja en cada caso indefinido el objeto del consenso sobre una rango prácticamente infinito de normas, de modo que el consenso básico se limite a los principios. Estos ofrecerán, luego, el marco procedimental a lo largo de los cuales se puede, luego, obtener consensos legítimos más específicos. A la inversa, al delimitar un sistema de esta clase, se hace visible qué alcance objetivo puede tener el consenso y qué queda, inevitablemente, fuera de él.

Los dos principios sobre los que, a mi modo de ver, se puede fundamentar normativamente un régimen democrático, son los siguientes:

I. Convengamos en que ningún miembro de las sociedad matará nunca a cualquier otro miembro de ella, ni le infligirá tormentos, ni pretenderá mediante la aplicación de coacciones físicas o psíquicas un asentimiento forzado para la satisfacción de sus propios fines.

II. Convengamos en que todo miembro de la sociedad tendrá siempre iguales prerrogativas que cualquier otro miembro de ella.

I es el principio de la dignidad de la persona bumana que, hasta donde alcanzo a ver, es equivalente en sus consecuencias a la tercera formulación del imperativo kantiano.

II es el principio de igualdad de las personas o, simplemente, el principio de igualdad numérica entre los miembro de una sociedad y es equivalente a los principios similares sostenidos por L. Nelson y J. Rawls.

Veamos, ahora, algunos aspectos del funcionamiento de estos dos principios.

(i) Como indiqué en general con relación a los actos asociativos, su validez depende de la aceptación o el rechazo por parte del oyente de la exhortación asociativa del hablante. Mientras que la aceptación, dado el aspecto directivo y compromisivo a un tiempo, implica validez inmediata, es decir, vigencia efectiva del contenido proposicional propuesto, que genera obligación moral de ambas partes, el rechazo deja a la exhortación suspendida y equivale a su retiro, a su anulación, a todos sus efectos. Ahora bien, si observamos el contenido proposicional de los dos principios, inmediatamente se ve que tienen ambos un cuantificador universal (ninguno, todos) y un operador temporal (nunca, 
siempre). En virtud de estas constantes lógicas, el rechazo de cualquiera de los principios no es, sin embargo, neutro, sino que equivale a 'convenir' la proposición contradictoria correspondiente, que se puede formular así: I' 'Hemos convenido que algún miembro de la sociedad matará, etc. en algún momento a otro miembro cualquiera de ella', II' 'Hemos convenido que algún miembro de la sociedad no tendrá algunas veces iguales prerrogativas que cualquier otro'. De este modo, se invierte la dirección argumentativa de los contractualistas al estilo de Hobbes: el estado de naturaleza no es la situación originaria, sino que proviene de la negación contradictoria a una exhortación universal y constituye, también, una forma de convención: la que da validez a la lucha a muerte.

(ii) Si prestamos atención a la formulación de los principios, veremos que I es negativo, mientras que II es positivo. A la manera de Rawls, creo que hay un orden indexical entre I y II, que me parece evidente de por sí. Esto está de acuerdo, a su vez, con el carácter general que, a mi juicio, tienen las normas morales: expresan restricciones.

En el principio de la dignidad humana se fundamentan las probibiciones de interferir violenta o coactivamente en las acciones de los otros, comenzando por el respeto por su vida. Sobre este principio se apoya, en el ámbito jurídico, el derecho penal. De este modo, el principio da fundamento 2 los dos ámbitos principales de la moral privada y de la moral pública. Dicho de otro modo, ningún consenso fáctico posterior podrá transgredir nunca este principio fundamental, ya que todo consenso lo tiene como su fundamento irreversible.

De II, en cambio, cuya formulación es positiva, surgen las reglas constitutivas del juego democrático, comenzando por el establecimiento de una constitución como marco formal fijo que establezca los procedimientos de elección de las autoridades, de promulgación de las normas que regulen el funcionamiento de la sociedad y permitan a sus miembros contraer obligaciones mutuas sobre la base de contratos libremente admitidos y que actúan en ambas direcciones. Nuevamente, el principio establece aquí una restricción a estos convenios, limitando su validez a la vigencia de la igualdad efectiva de las personas. Dado que es un postulado de esta fundamentación discursiva la participación incondicional de todos los miembros del sistema político, por sí o por no, en la aceptación de las reglas fundacionales, de la aceptación de II surge como un corolario una forma discursiva del principio rawlsiano de la diferencia o del principio nelsoniano del verdadero interés. Se puede formular así: III puesto que nadie es un guardián más celoso de sus propias prerrogativas que él mismo y dado que el respeto a los principios I y II exige que cada uno defienda sus derechos discursivamente, todos deben tener iguales posibilidades de alcanzar capacidad madura para comprender sus derechos y articular discursivamente sus demandas. 
7. Quisiera concluir indicando qué queda dentro del límite normativo y qué fuera. En términos generales se pueden dividir los intentos fundacionales en dos grandes grupos: 1) uno que asume la existencia de modos y formas de vida previos, históricos, culturales y sociales, de los miembros de una sociedad, quienes eventualmente pueden integrar un régimen u otro, de acuerdo con un conjunto posible de características (el gran modelo de esta concepción sigue siendo Aristóteles); 2) otro que establece un punto de partida fundacional, ante quo no existen ni derechos ni garantías, ya que éstas emanan de esa única fuente: el pacto. A mi modo de ver, la propuesta discursiva que he lanzado media entre ambas posiciones, ya que el convenio del cual emergen los dos principios no es un convenio fundacional de todos los rasgos éticamente relevantes de los miembros, ni siquiera de aquél carácter más elemental: la libertad de los miembros. En efecto, ésta es propuesta como previamente existente, ya que está latente en la posibilidad de rechazo. Los principios, que son la base de todo deber ser, no tienen dominio sobre esa libertad, que es un factum.

Pero también deja fuera otros dos hechos sumamente importantes para la constitución y el desarrollo de la democracia: por un lado, las condiciones naturales, el suelo, el clima, la constitución física de los hombres y mujeres, la capacidad técnica acumulada, todo aquello, en fin, que con Aristóteles podemos designar como ta anankaia o la escasez moderada de Rawls. Por el otro, otro factum tan importante como el anterior: las tradiciones culturales. En efecto, creo que normalmente no se toma lo suficientemente en cuenta la importancia y densidad de las herencias históricas. A mi modo de ver, la contribución destacable de los neoaristotélicos desde J. Ritter a A. MacIntyre, ha sido y es el destacar la importancia de esta herencia ambigua y refractaria a generalizaciones teóricas. Pues en los repliegues de esta tradición se depositan los valores positivos, estéticos-prácticos, que conforman los ideales históricos de vida y promueven la integración autorrealizativa de las personalidades, pero también las situaciones solapadas de poder, la conglomeración corporativista de los intereses y las idelogías distorsionadoras que apuntan hacia la obnubilación de la madura capacidad discursiva de los actores sociales. Es de aquí de donde, en todo momento, surge el impulso corrosivo del marco normativo. En efecto, éste, a pesar de ser lógicamente prioritario, debe abrirse camino, como una tradición más entre otras tradiciones, en ese mundo conflictivo y muchas veces opaco a formas discursivas de razón. Esto es particularmente visible allí donde la democracia está en estado llamado de transición. En efecto, dado que la libertad de actuar contra-consensualmente puede ser reasumida en cualquier momento por los miembros, instaurando de hecho el estado de lucha sin cuartel, la única posibilidad de la democracia como marco normativo es, paradójicamente, su corporización como tradición de un modo de actuar hacia el futuro por parte de los ciudadanos. No se trata sólo de las 'venta- 
jas de la democracia', sino de las convicciones con relación al futuro de los miembros de una sociedad y de la incorporación de esas convicciones en los proyectos individuales y comunitarios de vida como, justamente, proyectos de vida-en-una-sociedad-democrática.

No hay como haber vivido y vivir en una sociedad - la argentina- que desde hace medio siglo está siempre al borde de la quiebra de la democracia como para tomar conciencia del aspecto de promesa hacia el futuro que este régimen conlleva. Más que en las instituciones mismas, más que en un pacto pasado o en la vigencia de un determinado orden jurídico cuyos mecanismos hayan sido probados múltiples veces, la democracia se funda, en última instancia, en la disposición convencida de cada uno de sus miembros de que su acción futura se desarrollará de acuerdo con las normas que rigen el presente democrático. Es, paradójicamente, esta convicción presente sobre la preservación futura de este mismo estado de relaciones entre sus miembros, convicción compartida por la inmensa mayoría de ellos, lo que consolida la persistencia de una estado democrático. La tarea crítica de una fundamentación discursiva de la democracia como la expuesta, se inserta, a mi juicio, precisamente en esta coyuntura.

\section{REFERENCIAS}

Botana, N., La tradición republicana, Sudamericana, Buenos Aires, 1984.

Guariglia, O. N., Ideologia, verdad y legitimación, Sudamericana, Buenos Aires, 1986 $=I V L$.

- "Problemas conceptuales de la democracia", Revista de filosofia y teorla politica (La Plata), No. 26-27 (1986), pp. 29-36 = PCD.

- "Razón práctica e intereses de la acción", Ditinoia, 33 (1987), pp. 53-68.

Habermas, J., Theoric des kommunikativen Handelns, Suhrkamp, Francfort del Main, $1981=T K H$.

Kant, I., Grundlegung zur Metaphysik der Sitten, Werke, W. Weischedel (ed.), WB, Darmstad, 1966, tomo IV = GMS.

Macpherson, C. B., Democratic Theory, Clarendon Press, Oxford, 1973.

Marx, K., Werke, H.J. Lieber y P. Furth (eds.), WB, Darmstadt, 1962 y ss., 6 tomos. Mill, J. Stuart, On Liberty, en Utilitarianism, M. Warnock (ed.), Collins, Glasgow, 1979 (la. ed., 1859).

- Considerations on Representative Government, Routledge, Londres, s.f (la. ed., 1861).

$\longrightarrow$, Autobiografía, Espasa Calpe, Buenos Aires, 1939 (la. ed., 1873).

Pateman, C., Participation and Democratic Theory, Cambridge University Press, Cambridge, 1970.

Riley, J., "On the Possibility or Liberal Democracy", Amer. Poli. Sc. Rev. 79 (1985), pp. 1135-1151. 
Searle, J. R., "A Taxonomy of Ilocutionary Acts", en Expression and Meaning, Cambridge University Press, Cambridge, 1979, pp. 1-30 =LA.

Schumpeter, J. A., Capitalism, Socialism and Democracy, G. Allen and Unwin, Londres, 4a. ed., 1952 (la. ed., 1943). 